\title{
On the semiadditivity of analytic capacity and planar Cantor sets
}

\author{
Joan Mateu, Xavier Tolsa, and Joan Verdera
}

\begin{abstract}
It has been recently proved that analytic capacity, $\gamma$, is semiadditive. This result is a consequence of the comparability between $\gamma$ and $\gamma_{+}$, a version of $\gamma$ originated by bounded Cauchy potentials of positive measures. In this paper we describe the main ideas involved in the proof of this result and we give a complete proof of it in the particular case of the $N$-th approximation of the corner quarters Cantor set.
\end{abstract}

\section{Introduction}

There has been recently some progress in connection with the theory of analytic capacity. The main result, obtained by the second author [To4], asserts that analytic capacity $\gamma$ is semiadditive, that is,

$$
\gamma(E \cup F) \leq C(\gamma(E)+\gamma(F))
$$

for all compact sets $E, F \subset \mathbb{C}$, where $C$ is an absolute constant. This confirms a 35 years old conjecture of Vitushkin [Vi] and, consequently, provides new criteria for uniform rational approximation.

The semiadditivity inequality above is derived in [To4] from another fact of independent interest, namely that analytic capacity can be defined, modulo constants, by means of bounded Cauchy potentials of positive measures. In other words, there exists a positive number $\mathrm{C}$ such that

$$
\gamma(E) \leq C \gamma_{+}(E)
$$

for all compact subsets $\mathrm{E}$ of the plane (see below for precise definitions).

In [MTV] we had already obtained inequality (1.1) for some planar Cantor sets. This was used there to determine the Cantor sets of zero analytic capacity, which solved a long standing open problem raised by Garnett in [Gar2].

The authors were partially supported by grants BFM2000-0361, HPRN-2000-0116, and 2001-SGR-00431. Xavier Tolsa was supported also by a Marie Curie Fellowship of the European Community program Human Potential under contract HPMFCT-2000-00519 and by the program Ramón y Cajal, MCYT (Spain). 
The goal of this expository paper is to explain the main ideas and techniques used in the proof of the results mentioned above. In Section 3 we present a complete proof of (1.1) in the particular case in which $E=E_{N}$, the N-th approximation of the familiar corner quarters Cantor set. In this setting the technical complications are reduced to a minimum and thus the main ideas emerge easily. In Section 4 we describe the new difficulties that arise in the proof of (1.1) for general compact sets and the new ideas required to overcome them. The applications of semiadditivity to rational and harmonic approximation are presented in Section 5. In the last section the reader will find a short list of open problems that we think are interesting and worth working at.

Before formulating the results in detail, we will recall some definitions. The analytic capacity of a compact set $E \subset \mathbb{C}$ is defined as

$$
\gamma(E)=\sup \left|f^{\prime}(\infty)\right|
$$

where the supremum is taken over all analytic functions $f: \mathbb{C} \backslash E \longrightarrow \mathbb{C}$ with $|f| \leq 1$ on $\mathbb{C} \backslash E$ and $f^{\prime}(\infty)=\lim _{z \rightarrow \infty} z(f(z)-f(\infty))$. For a general set $F$, we set $\gamma(F)=\sup \{\gamma(E): E \subset F$, E compact $\}$.

The notion of analytic capacity was first introduced by Ahlfors [Ah] in the 1940's in order to study the removability of singularities of bounded analytic functions. A compact set $E \subset \mathbb{C}$ is said to be removable (for bounded analytic functions) if for any open set $\Omega$ containing $E$, every bounded function analytic on $\Omega \backslash E$ has an analytic extension to $\Omega$. Ahlfors remarked that $E$ is removable if and only if $\gamma(E)=0$.

In the 1950's, Vitushkin showed that analytic capacity plays a fundamental role in problems of uniform approximation of analytic functions by rational functions (see [Vi] , for example). The main drawback of Vitushkin's results arises from the fact that there is not a complete description of analytic capacity in metric or geometric terms.

The capacity $\gamma_{+}$(or analytic capacity $\gamma_{+}$) of a compact set $E$ is

$$
\gamma_{+}(E)=\sup _{\mu} \mu(E)
$$

where the supremum is taken over all positive Radon measures $\mu$ supported on $E$ such that the Cauchy potential $f=\frac{1}{z} * \mu$ is an $L^{\infty}(\mathbb{C})$ function with $\|f\|_{\infty} \leq 1$. Since $\left(\frac{1}{z} * \mu\right)^{\prime}(\infty)=\mu(E)$, we have

$$
\gamma_{+}(E) \leq \gamma(E) \text {. }
$$

For a general set $F$, we set $\gamma_{+}(F)=\sup \left\{\gamma_{+}(E): E \subset F\right.$, E compact $\}$.

In [To4] it was shown that the converse of inequality (1.2) (modulo a multiplicative constant) also holds:

THEOREM 1.1. There exists an absolute constant $C$ such that

$$
\gamma(E) \leq C \gamma_{+}(E)
$$

for any compact set $E$. 
Therefore, we get $\gamma(E) \approx \gamma_{+}(E)$ (where $a \approx b$ means that there exists an absolute positive constant $C$ such that $C^{-1} b \leq a \leq C b$ ), which solves an old question about analytic capacity (see for example [DØ] or [Ve1, p.435]).

Since the capacity $\gamma_{+}$is countably semiadditive (see [To2]), the preceding theorem implies that $\gamma$ is also countably semiadditive:

Theorem 1.2. Let $E_{n}, n=1,2, \ldots$, be Borel sets. Then,

$$
\gamma\left(\bigcup_{n} E_{n}\right) \leq C \sum_{n} \gamma\left(E_{n}\right)
$$

where $C$ is an absolute constant.

Let us mention another consequence of Theorem 1.1. As shown in [MV], there is a strong relationship between the $L^{2}$ boundedness of the Cauchy integral operator and the Menger curvature of measures (see Section 4 for this relationship and for the notion of Menger curvature of measures). As a consequence, it turns out that a compact set $E \subset \mathbb{C}$ is non removable for bounded analytic functions if and only if it supports a Radon measure $\mu$ with finite Menger curvature and such that $\mu(B(x, r)) \leq r$ for all $x \in \mathbb{C}, r>0$.

Now we turn our attention to Cantor sets. Given a sequence $\lambda=$ $\left\{\lambda_{n}\right\}_{n \geq 1}$, with $0 \leq \lambda_{n} \leq 1 / 3$, we construct a planar Cantor set as follows. Let $Q^{0}=[0,1] \times[0,1]$. At the first step we take four closed squares inside $Q^{0}$, with side length $\lambda_{1}$, with sides parallel to the coordinate axes, and so that each square contains a vertex of $Q^{0}$. At the second step we apply the preceding procedure to each of the four squares obtained in the first step, but now using the proportion factor $\lambda_{2}$. In this way, we get 16 squares of side length $\sigma_{2}=\lambda_{1} \lambda_{2}$. Proceeding inductively, at each step we obtain $4^{n}$ squares $Q_{j}^{n}, j=1, \ldots, 4^{n}$ with side length $\sigma_{n}=\lambda_{1} \cdots \lambda_{n}$. We denote

$$
E_{n}=E_{n}(\lambda)=\bigcup_{j=1}^{4^{n}} Q_{j}^{n}
$$

and we define

$$
E=E(\lambda)=\bigcap_{n=1}^{\infty} E_{n}
$$

If we set $\lambda_{n}=1 / 4$ for all $n$, then $E(\lambda)$ is the so called "corner quarters Cantor set", which has positive and finite 1-dimensional Hausdorff measure but zero analytic capacity [Gar2].

The problem of estimating the analytic capacity of the Cantor sets $E(\lambda)$ was first considered by Garnett [Gar2]. Mattila [Ma] proved that if

$$
\sum_{n=1}^{\infty} \frac{1}{\left(4^{n} \sigma_{n}\right)^{2}}<\infty
$$


then $\gamma(E(\lambda))>0$. In fact, his arguments show that the following quantitative estimate holds

$$
\gamma_{+}\left(E_{N}\right) \geq C\left(\sum_{n=1}^{N} \frac{1}{\left(4^{n} \sigma_{n}\right)^{2}}\right)^{-1 / 2},
$$

where $C$ is some positive absolute constant. Eiderman $[\mathbf{E i}]$ proved the converse inequality:

$$
\gamma_{+}\left(E_{N}\right) \leq C\left(\sum_{n=1}^{N} \frac{1}{\left(4^{n} \sigma_{n}\right)^{2}}\right)^{-1 / 2}
$$

(see also [To3] for a different proof). In [MTV] it was shown that $\gamma\left(E_{N}\right) \approx$ $\gamma_{+}\left(E_{N}\right)$, with absolute constants. So we get the following result.

TheOrem 1.3. For all $N \geq 1$, we have

$$
\gamma\left(E_{N}\right) \approx\left(\sum_{n=1}^{N} \frac{1}{\left(4^{n} \sigma_{n}\right)^{2}}\right)^{-1 / 2} .
$$

As a consequence, the Cantor set $E(\lambda)$ has positive analytic capacity if and only if (1.3) holds.

Taking $\lambda_{n}=1 / 4$, for all $n$, we obtain the following.

COROLlary 1.4. If $E_{N}$ is the $N$-th approximation of the corner quarters Cantor set, then

$$
\gamma\left(E_{N}\right) \approx N^{-1 / 2}
$$

\section{Preliminaries}

Given a complex Radon measure $\nu$ on $\mathbb{C}$, the Cauchy transform of $\nu$ is

$$
\mathcal{C} \nu(z)=\int \frac{1}{\xi-z} d \nu(\xi)
$$

This definition does not make sense, in general, for $z \in \operatorname{supp}(\nu)$, although one can easily see that the integral above is absolutely convergent at a.e. $z \in \mathbb{C}$ (with respect to Lebesgue measure). This is the reason why one considers the truncated Cauchy transform of $\nu$, which is defined as

$$
\mathcal{C}_{\varepsilon} \nu(z)=\int_{|\xi-z|>\varepsilon} \frac{1}{\xi-z} d \nu(\xi)
$$

for any $\varepsilon>0$ and $z \in \mathbb{C}$. Given a $\mu$-measurable function $f$ on $\mathbb{C}$ (where $\mu$ is some fixed positive Radon measure on $\mathbb{C})$, we write $\mathcal{C} f:=\mathcal{C}(f d \mu)$ and $\mathcal{C}_{\varepsilon} f:=\mathcal{C}_{\varepsilon}(f d \mu)$ for any $\varepsilon>0$. It is said that the Cauchy transform is bounded on $L^{2}(\mu)$ if the operators $\mathcal{C}_{\varepsilon}$ are bounded on $L^{2}(\mu)$ uniformly on $\varepsilon>0$.

The maximal Cauchy transform of $\nu$ is defined as

$$
\mathcal{C}_{*} \nu(z)=\sup _{\varepsilon>0}\left|\mathcal{C}_{\varepsilon} \nu(z)\right| \text {. }
$$


As above, we set $\mathcal{C}_{*} f:=\mathcal{C}_{*}(f \mu)$.

A positive Radon measure $\mu$ is said to have linear growth if there exists some constant $C$ (depending on $\mu$ ) such that $\mu(B(x, r)) \leq C r$ for all $x \in \mathbb{C}$, $r>0$. Let us remark that, strictly speaking, one should say something like "linear $C$-growth" instead of linear growth.

Given three pairwise different points $x, y, z \in \mathbb{C}$, their Menger curvature is

$$
c(x, y, z)=\frac{1}{R(x, y, z)}
$$

where $R(x, y, z)$ is the radius of the circumference passing through $x, y, z$ (with $R(x, y, z)=\infty, c(x, y, z)=0$ if $x, y, z$ lie on a same line). If two among these points coincide, we let $c(x, y, z)=0$. For a positive Radon measure $\mu$, we set

$$
c_{\mu}^{2}(x)=\iint c(x, y, z)^{2} d \mu(y) d \mu(z),
$$

and we define the curvature of $\mu$ as

$$
c^{2}(\mu)=\int c_{\mu}^{2}(x) d \mu(x)=\iiint c(x, y, z)^{2} d \mu(x) d \mu(y) d \mu(z) .
$$

The $\varepsilon$-truncated version of $c^{2}(\mu)$, which we denote as $c_{\varepsilon}^{2}(\mu)$, is defined as in the right hand side of (2.1), but with the triple integral over $\{x, y, z \in \mathbb{C}$ : $|x-y|,|y-z|,|x-z|>\varepsilon\}$.

The notion of curvature of a measure, which was introduced by Melnikov [Me] when he was studying a discrete version of analytic capacity, is connected to the Cauchy transform. This relationship comes from the following identity found by Melnikov and Verdera [MV] (assuming that $\mu$ has linear growth):

$$
\left\|\mathcal{C}_{\varepsilon} \mu\right\|_{L^{2}(\mu)}^{2}=\frac{1}{6} c_{\varepsilon}^{2}(\mu)+O(\mu(\mathbb{C})),
$$

In [Me], Melnikov proved the following inequality:

$$
\gamma(E) \geq C \sup _{\mu \in \Sigma(E)} \frac{\mu(E)^{2}}{\mu(E)+c^{2}(\mu)},
$$

where $C>0$ is some absolute constant and $\Sigma(E)$ stands for the set of all positive Radon measures $\mu$ supported on $E \subset \mathbb{C}$ such that $\mu(B(x, r)) \leq r$ for all $x \in E, r>0$. In [To2] it was shown that inequality (2.3) also holds if one replaces $\gamma(E)$ by $\gamma_{+}(E)$ on the left hand side, and then one obtains

$$
\gamma_{+}(E) \approx \sup _{\mu \in \Sigma(E)} \frac{\mu(E)^{2}}{\mu(E)+c^{2}(\mu)} .
$$

Let $M$ be the maximal radial Hardy-Littlewood operator:

$$
M \mu(x)=\sup _{r>0} \frac{\mu(B(x, r))}{r} .
$$


The following potential was introduced in [Ve2]:

$$
U_{\mu}(x):=M \mu(x)+c_{\mu}(x),
$$

It turns out that $\gamma_{+}$can also be characterized in terms of this potential (see [To3], and also [Ve2] for a related result):

$$
\gamma_{+}(E) \approx \sup \left\{\mu(E): \operatorname{supp}(\mu) \subset E, U_{\mu}(x) \leq 1 \forall x \in E\right\} .
$$

Moreover, it has been shown in [To3] that $\gamma_{+}$(and thus $\gamma$ by Theorem 1.1) also satisfies the following inequality of weak type $(1,1)$ :

$$
\gamma_{+}\left\{x \in \mathbb{C}: U_{\mu}(x)>\lambda\right\} \leq \frac{C \mu(\mathbb{C})}{\lambda},
$$

for all $\lambda>0$. At this point it is worth to remark that $\gamma_{+}$(and thus $\gamma$ ) and the Cauchy transform are also connected by another inequality of weak type $(1,1)$ :

$$
\gamma_{+}\left\{x \in \mathbb{C}: \mathcal{C}_{*} \nu(x)>\lambda\right\} \leq \frac{C|\nu|(\mathbb{C})}{\lambda},
$$

for all $\lambda>0$ and any complex measure $\nu$ (see [To3]).

The reader can obtain more information on Menger curvature and its relationship with the $L^{2}$ boundedness of the Cauchy integral in the expository paper [Ve3].

By a square $Q$ we mean a closed square with sides parallel to the axes.

Throughout all the paper, the letter $C$ will stand for an absolute constant that may change at different occurrences. Constants with subscripts, such as $C_{1}$, will retain its value.

\section{The analytic capacity of the corner quarters Cantor set}

In this section we will prove Theorem 1.3 in the special case $\lambda_{n}=1 / 4$ for all $n$. So $E(\lambda)$ stands for the corner quarters Cantor set throughout all the section (and $E_{N}$ for its $N$-th generation).

3.1. Estimate of $\gamma_{+}\left(E_{N}\right)$. For the reader's convenience, we are going to explain how one can prove that $\gamma_{+}\left(E_{N}\right) \approx N^{-1 / 2}$. We will follow the approach in [To3, Section 3.1]. Assume $N \geq 2$ and consider the measure $\mu=d s_{\mid \partial E_{N}}$. Given $x \in E_{N}$, let $Q^{n}(x)$ be the square $Q_{j}^{n}, 1 \leq j \leq 4^{n}$, that contains $x$. We have

$$
\begin{aligned}
c_{\mu}^{2}(x) & \geq \sum_{n=1}^{N-1} \iint_{y, z \in Q^{n-1}(x) \backslash Q^{n}(x)} c(x, y, z)^{2} d \mu(y) d \mu(z) \\
& \geq C^{-1} \sum_{n=1}^{N-1} \frac{\mu\left(Q^{n}(x)\right)^{2}}{\ell\left(Q^{n}(x)\right)^{2}}=C^{-1}(N-1) \geq C^{-1} N .
\end{aligned}
$$

Thus, $U_{\mu}(x) \geq C^{-1} N^{1 / 2}$ for all $x \in E_{N}$. From (2.7), we deduce $\gamma_{+}\left(E_{N}\right) \leq$ $C \mu\left(E_{N}\right) N^{-1 / 2}=C N^{-1 / 2}$. 
With little additional effort the reader can also verify that there exists some absolute constant $C$ such that $c_{\mu}^{2}(x) \leq C N$ for all $x \in E_{N}$. So $U_{\mu}(x) \leq$ $C N^{1 / 2}$ and then, by $(2.6), \gamma_{+}\left(E_{N}\right) \geq C^{-1} N^{1 / 2}$.

3.2. The local $T(b)$ theorem of Christ. The fundamental tool to prove Theorem 1.3 (in the case $\lambda_{n}=1 / 4$ ) will be the local $T(b)$ Theorem of Christ [Ch1], which is valid for general Calderón-Zygmund operators in the setting of spaces of homogeneous type (see $[\mathbf{C W}]$ ). However, we will state it for the particular case of the Cauchy transform on the set $\partial E_{N}$ and a measure of the form $\mu=c d s_{\mid \partial E_{N}}$, with $c \leq 2$.

THEOREM 3.1 (Christ). Consider the measure $\mu=c d s_{\mid \partial E_{N}}$, with $c \leq 2$. Suppose that for each square $Q_{j}^{n}, n=0, \ldots, N, j=1, \ldots, 4^{n}$, there exists a function $b_{j}^{n} \in L^{\infty}(\mu)$, supported on $Q_{j}^{n}$, such that

(a) $\left\|b_{j}^{n}\right\|_{\infty} \leq C_{1}$,

(b) $\left\|\mathcal{C}_{\varepsilon}\left(b_{j}^{n}\right)\right\|_{\infty} \leq C_{1}$ uniformly on $\varepsilon>0$,

(c) $\mu\left(Q_{j}^{n}\right) \leq C_{1}\left|\int b_{j}^{n} d \mu\right|$.

Then, the Cauchy transform is bounded on $L^{2}(\mu)$ with norm bounded above by some constant $C_{2}$ which depends only on $C_{1}$.

Below, we will use Theorem 3.1 to show that the Cauchy transform is bounded on $L^{2}(\mu)$ with norm bounded above by an absolute constant. The assumption $c \leq 2$ is a convenient normalization which ensures that $C_{2}$ does not depend on $c$.

Notice that the measure $\mu$ above has linear growth and is doubling (both conditions with absolute constants, independent of $c$ ). On the other hand, it is clear that the constant $a$ in the inequality $\mu(B(x, r)) \geq a r, x \in E_{N}$, $0<r \leq 1$, is not absolute since it is essentially a multiple of $c$. However, this does not cause any trouble because Christ's theorem depends only on the doubling and linearity constants.

3.3. Some preliminary comments. In order to show that

$$
\gamma\left(E_{N}\right) \leq C \gamma_{+}\left(E_{N}\right)
$$

we would like to prove that the Cauchy transform is bounded on $L^{2}(\mu)$, where $\mu=\frac{1}{4} \gamma\left(E_{N}\right) d s_{\mid \partial E_{N}}$, with the $L^{2}(\mu)$ norm bounded above by some absolute constant. Then, by a dualization argument of the weak $(1,1)$ inequality (see [Ch2, p. 107], for example), it follows that

$$
\gamma_{+}\left(E_{N}\right) \geq C^{-1} \mu\left(E_{N}\right)
$$

which clearly implies $(3.1)$, since $\mu\left(E_{N}\right)=\gamma\left(E_{N}\right)$.

From the $L^{2}(\mu)$ boundedness of the Cauchy transform, one can also derive (3.2) via an estimate of the curvature $c^{2}(\mu)$ by means of (2.2), and the characterization of $\gamma_{+}$in terms of curvature in (2.4) [in this case the dualization argument for the weak $(1,1)$ inequality is contained in the proof of the estimate (2.4)]. 
Because of the definition of analytic capacity, we know that there exists a function $f$ (the Ahlfors function) analytic in $\mathbb{C} \backslash E_{N}$ such that $|f(z)| \leq 1$ in $\mathbb{C} \backslash E_{N}$ and $f^{\prime}(\infty)=\gamma\left(E_{N}\right)$. Let $b$ be the function supported on $\partial E_{N}$ defined by

$$
\frac{1}{2 \pi i} f(z) d z_{\mid \partial E_{N}}=b(z) d \mu(z),
$$

where $f(z)$ stands for the outside boundary values of $f$ at $\partial E_{N}$. Then $b$ satisfies the following conditions:

$$
\begin{gathered}
\|b\|_{\infty} \leq \frac{2}{\pi \gamma\left(E_{N}\right)}, \\
|\mathcal{C}(b d \mu)(z)| \leq 1 \quad \text { for } z \notin E_{N},
\end{gathered}
$$

and

$$
\mu\left(E_{N}\right)=\int b d \mu .
$$

By standard arguments, it easy to check that (3.4) implies that

$$
\left|\mathcal{C}_{\varepsilon}(b d \mu)(z)\right| \leq C \quad \text { for } z \in E_{N} \text { and all } \varepsilon>0,
$$

Let us see what happens if we apply directly the local $T(b)$ Theorem of Christ in $L^{2}(\mu)$ using the function $b$. We consider only the square $Q_{j}^{n}=Q^{0}$. We must show that there exists a function $b^{0}$ supported on $\partial E_{N}$ satisfying (a), (b) and (c) of Theorem 3.1. If we set $b^{0}=b$, then (b) follows from (3.6), and (c) from (3.5). However, we cannot deduce (a) from (3.3), since it may happen $\gamma\left(E_{N}\right) \rightarrow 0$ as $N \rightarrow \infty$ (in fact, it happens).

The argument above fails because we have a very bad estimate for $\|b\|_{\infty}$. By an elementary localization argument (see [Gar1, Lemma 1, (b)]), we can obtain the following additional information on $b$ :

$$
\left|\int_{Q_{j}^{n}} b d \mu\right| \leq C \gamma\left(Q_{j}^{n} \cap E_{N}\right) \quad \text { for } n=0, \ldots, N, j=1, \ldots, 4^{n} .
$$

Notice that if $b$ were bounded by an absolute constant, then we would have

$$
\left|\int_{Q_{j}^{n}} b d \mu\right| \leq C \mu\left(Q_{j}^{n}\right) \quad \text { for } n=0, \ldots, N, j=1, \ldots, 4^{n} .
$$

In fact, it is easy to see that if the latter estimate were true, then we would be able to construct a (slight) variant of $b$ satisfying (a), (b) and (c) in Theorem 3.1, for $Q^{0}$.

So the following question arises naturally. For which squares $Q_{j}^{n}$ is it true that

$$
\gamma\left(Q_{j}^{n} \cap E_{N}\right) \leq C \mu\left(Q_{j}^{n}\right) ?
$$


This condition clearly holds for $Q_{j}^{n}=Q^{0}$, but it is false for the small squares $Q_{j}^{N}$, since $\gamma\left(Q_{j}^{N} \cap E_{N}\right) \approx \ell\left(Q_{j}^{N}\right)$ but $\mu\left(Q_{j}^{N}\right)=\gamma\left(E_{N}\right) \ell\left(Q_{j}^{N}\right)$. For the intermediate squares $Q_{j}^{N / 2}$ (assuming $N$ even) we have

$$
\gamma\left(Q_{j}^{N / 2} \cap E_{N}\right)=\ell\left(Q_{j}^{N / 2}\right) \gamma\left(E_{N / 2}\right),
$$

since $Q_{j}^{N / 2} \cap E_{N}$ coincides with a dilation of $E_{N / 2}$ with factor $\ell\left(Q_{j}^{N / 2}\right)$. Also,

$$
\mu\left(Q_{j}^{N / 2}\right)=\ell\left(Q_{j}^{N / 2}\right) \gamma\left(E_{N}\right) .
$$

Therefore, for these squares (3.9) is equivalent to

$$
\gamma\left(E_{N / 2}\right) \leq C \gamma\left(E_{N}\right)
$$

Notice also that this estimate is a necessary condition for $\gamma\left(E_{N}\right) \approx N^{-1 / 2}$. In next subsection, arguing by induction, we will see that we can always assume (3.10) to be true.

\subsection{Proof of Theorem $\mathbf{1 . 3}$ for the corner quarters Cantor set.} We will show that $\gamma\left(E_{N}\right) \leq C_{3} N^{-1 / 2}$ by induction on $N$, where $C_{3}$ is some fixed constant that will be fixed below. The cases $N=0,1$ are trivial. Indeed, we only have to choose $C_{3}$ big enough. Now we will prove that if

$$
\gamma\left(E_{M}\right) \leq \frac{C_{3}}{M^{1 / 2}} \quad \text { for } M \leq N / 2,
$$

then this also holds for $M=N$. We denote $M_{0}=N / 2$ if $N$ is even, and $M_{0}=(N-1) / 2$ if $N$ is odd. We distinguish two cases:

(1) $\gamma\left(E_{M_{0}}\right) \leq 2 \gamma\left(E_{N}\right)$.

(2) $\gamma\left(E_{M_{0}}\right)>2 \gamma\left(E_{N}\right)$.

In Case (2), we deduce

$$
\gamma\left(E_{N}\right) \leq \frac{1}{2} \gamma\left(E_{M_{0}}\right) \leq \frac{C_{3}}{\left(4 M_{0}\right)^{1 / 2}} \leq \frac{C_{3}}{[2(N-1)]^{1 / 2}} \leq \frac{C_{3}}{N^{1 / 2}} .
$$

So we are left with Case (1). We take the measure

$$
d \mu=\frac{1}{4} \gamma\left(E_{N}\right) d s_{\mid \partial E_{M_{0}}}
$$

(notice the difference with the measure $\mu$ in the preceding subsection). Our goal is to show that the Cauchy transform is bounded on $L^{2}(\mu)$ with absolute constants. Since $\mu$ is supported on $E_{M_{0}}$, this would imply that

$$
\gamma_{+}\left(E_{M_{0}}\right) \geq C^{-1} \mu\left(E_{M_{0}}\right)=C^{-1} \gamma\left(E_{N}\right),
$$

and so, by Eiderman's inequality (1.5),

$$
\gamma\left(E_{N}\right) \leq C \gamma_{+}\left(E_{M_{0}}\right) \leq \frac{C}{(N / 2)^{1 / 2}}=: \frac{C_{3}}{N^{1 / 2}},
$$

and the theorem would follow.

To verify that the Cauchy transform is bounded on $L^{2}(\mu)$, we will use the local $T(b)$ Theorem of Christ. Let $f$ be the Ahlfors function of $E_{N}$. Then $f$ 
is analytic on $\mathbb{C} \backslash E_{N},|f(z)| \leq 1$ for $z \notin E_{N}, f(\infty)=0$ and $f^{\prime}(\infty)=\gamma\left(E_{N}\right)$. Let $\nu$ be the complex measure $d \nu=\frac{1}{2 \pi i} f(z) d z \mid \partial E_{N}$, so that $\mathcal{C} \nu(z)=f(z)$ for $z \notin E_{N}$ and $\mathcal{C} \nu(z)=0$ if $z \in E_{N}$. Let $n$, with $0 \leq n \leq M_{0}$, be fixed. There exists an index $k, 1 \leq k \leq 4^{n}$, such that

$$
\gamma\left(E_{N}\right)=\sum_{j=1}^{4^{n}} \nu\left(Q_{j}^{n}\right) \leq 4^{n}\left|\nu\left(Q_{k}^{n}\right)\right| .
$$

This is equivalent to saying that $\mu\left(Q_{j}^{n}\right) \leq\left|\nu\left(Q_{k}^{n}\right)\right|$.

Now we will define the function $b_{k}^{n}$ associated to $Q_{k}^{n}$. First we need to introduce some auxiliary functions $\varphi$ and $\varphi_{j}, j=1, \ldots, 4^{M_{0}}$. We let $\varphi$ be a compactly supported $\mathcal{C}^{\infty}$ function on $\mathbb{C}$, with $0 \leq \varphi \leq 1$, such that $\int_{\partial Q^{0}} \varphi d s \geq 1$ and $\varphi$ vanishes on $\bigcup_{j=1}^{4} B\left(z_{j}, 1 / 4\right)$, where $z_{j}$ are the vertices of $Q^{0}$. Then, for $1 \leq j \leq 4^{M_{0}}$, we set

$$
\varphi_{j}(z)=\varphi\left(\frac{z-v_{j}}{4^{-M_{0}}}\right) \chi_{Q_{j}^{M_{0}}},
$$

where $v_{j}$ is the left lower vertex of $Q_{j}^{M_{0}}$. It is easy to see that $\left|\mathcal{C}_{\varepsilon}(\varphi d \mu)\right| \leq C$, and also that

$$
\int \varphi_{j} d \mu \geq \frac{1}{4} \mu\left(Q_{j}^{M_{0}}\right)
$$

We define

$$
b=b_{k}^{n}:=\sum_{Q_{j}^{M_{0}} \subset Q_{k}^{n}} \nu\left(Q_{j}^{M_{0}}\right) \frac{\varphi_{j}}{\int \varphi_{j} d \mu} .
$$

We clearly have

$$
\left|\int b d \mu\right|=\left|\nu\left(Q_{k}^{n}\right)\right| \geq \mu\left(Q_{k}^{n}\right) .
$$

To see that $b$ is bounded it suffices to show that

$$
\left|\nu\left(Q_{j}^{M_{0}}\right)\right| \leq C \mu\left(Q_{j}^{M_{0}}\right) \quad \text { for } 1 \leq j \leq 4^{M_{0}} .
$$

From a localization argument such as the one mentioned in (3.7), we get

$$
\left|\nu\left(Q_{j}^{M_{0}}\right)\right| \leq C \gamma\left(Q_{j}^{M_{0}} \cap E_{N}\right) .
$$

Suppose first that $N$ is even and $M_{0}=N / 2$. Then $Q_{j}^{M_{0}} \cap E_{N}$ coincides with a dilation of $E_{N / 2}$ by the factor $\ell\left(Q_{j}^{M_{0}}\right)$. Thus, using condition (1),

$$
\gamma\left(Q_{j}^{M_{0}} \cap E_{N}\right)=\ell\left(Q_{j}^{M_{0}}\right) \gamma\left(E_{N / 2}\right) \leq 4^{1 / 2} \ell\left(Q_{j}^{M_{0}}\right) \gamma\left(E_{N}\right)=4^{1 / 2} \mu\left(Q_{j}^{M_{0}}\right),
$$

and (3.11) follows. If $M_{0}$ is odd and $M_{0}=(N-1) / 2$, then $Q_{j}^{M_{0}} \cap E_{N}$ coincides with a dilation of $E_{(N+1) / 2}$ by the factor $\ell\left(Q_{j}^{M_{0}}\right)$. Thus, by the assumption (1) and the fact that $E_{(N+1) / 2} \subset E_{(N-1) / 2}=E_{M_{0}}$, we obtain

$$
\gamma\left(Q_{j}^{M_{0}} \cap E_{N}\right)=\ell\left(Q_{j}^{M_{0}}\right) \gamma\left(E_{(N+1) / 2}\right) \leq 4^{1 / 2} \ell\left(Q_{j}^{M_{0}}\right) \gamma\left(E_{N}\right)=4^{1 / 2} \mu\left(Q_{j}^{M_{0}}\right),
$$

and (3.11) also holds in this case. 
Let us check that $\mathcal{C}_{\varepsilon}(b d \mu)$ is a bounded function. Since $\left|\mathcal{C}_{\varepsilon}\left(\chi_{Q_{k}^{n}} d \nu\right)\right| \leq C$, we only have to prove that

$$
\left|\mathcal{C}_{\varepsilon}(b d \mu)-\mathcal{C}_{\varepsilon}\left(\chi Q_{k}^{n} d \nu\right)\right| \leq C .
$$

This follows by standard estimates, such as in [MTV, Section 3, (15)] (the reader should think that $b d \mu$ is an approximation of $\chi_{Q_{k}^{n}} d \nu$ at the level of the squares $Q_{j}^{M_{0}}$, and then $\mathcal{C}_{\varepsilon}(b d \mu)$ approximates $\left.\mathcal{C}_{\varepsilon}\left(\chi_{Q_{k}^{n}} d \nu\right)\right)$.

This completes the arguments required for the function $b_{k}^{n}$ associated to the square $Q_{k}^{n}$ in the local $T(b)$ Theorem.

Let us consider now the squares $Q_{j}^{n}$ of the $n$-th generation with $j \neq k$. We construct $b_{j}^{n}$ simply by translating $b_{k}^{n}$. We have $Q_{j}^{n}=w_{j}^{n}+Q_{k}^{n}$, for some $w_{j}^{n} \in \mathbb{C}$. We define $b_{j}^{n}(z)=b_{k}^{n}\left(z-w_{j}^{n}\right)$. It is clear that $b_{j}^{n}$ satisfies all the necessary assumptions to apply the local $T(b)$ Theorem.

Thus we have shown that the local $T(b)$ Theorem of Christ can be applied to $\mu$, and so the Cauchy transform is bounded on $L^{2}(\mu)$ with norm bounded above by some absolute constant. We are done.

\section{Some ideas for the proof of the general case}

In this section we will sketch the arguments involved in the proof of Theorem 1.1. First we will describe the similarities with the proof for the corner quarters Cantor set, and then we will give the new ideas for the general case.

By a standard discretization argument, such as the one in [Me, Lemma 1], it is enough to prove Theorem 1.1 assuming that $E$ is a finite union of compact disjoint segments.

As in the case of the corner quarters Cantor set, we want to apply some kind of $T(b)$ theorem, in the spirit of the local $T(b)$ Theorem of Christ. Because of the definition of analytic capacity, there exists a complex Radon measure $\nu_{0}$ supported on $E$ such that

$$
\begin{aligned}
\left\|\mathcal{C} \nu_{0}\right\|_{\infty} & \leq 1 \\
\left|\nu_{0}(E)\right| & =\gamma(E), \\
d \nu_{0} & =b_{0} d \mathcal{H}^{1} \mid E, \quad \text { with }\left\|b_{0}\right\|_{\infty} \leq 1 .
\end{aligned}
$$

We would like to show that there exists some Radon measure $\mu$ supported on $E$ with $\mu \in \Sigma(E), \mu(E) \approx \gamma(E)$, and such that the Cauchy transform is bounded on $L^{2}(\mu)$ with absolute constants. Then, as in the preceding section, we would get

$$
\gamma_{+}(E) \geq C^{-1} \mu(E) \geq C^{-1} \gamma(E),
$$

and we would be done.

However, by a more or less direct application of a $T(b)$ type theorem we cannot expect to prove that the Cauchy transform is bounded with respect to such a measure $\mu$ with absolute constants. Let us explain the reasons in some detail. Suppose for example that there exists some function $b$ such 
that $d \nu_{0}=b d \mu$ and we use the information about $\nu_{0}$ given by (4.1), (4.2) and (4.3). From (4.1) and (4.2) we derive

$$
\|\mathcal{C}(b d \mu)\|_{\infty} \leq 1
$$

and

$$
\left|\int b d \mu\right| \approx \mu(E)
$$

The estimate (4.4) is very good for our purposes. In fact, most classical $T(b)$ type theorems require only the $B M O(\mu)$ norm of $b$ to be bounded, which is a weaker assumption. The estimate (4.5) is likewise good; it is a global paraaccretivity condition, and with some technical difficulties (which may involve some kind of stopping time argument, like in [Ch1], [Da] or [NTV]), one can hope to be able to prove that the local paraaccretivity condition

$$
\left|\int_{Q} b d \mu\right| \approx \mu(Q \cap E)
$$

holds for many squares $Q$.

Our problems arise from (4.3). Notice that this implies that $\left|\nu_{0}\right|(E) \leq$ $\mathcal{H}^{1}(E)$, where $\left|\nu_{0}\right|$ stands for the variation of $\nu_{0}$. This is a very bad estimate since we don't have any control on $\mathcal{H}^{1}(E)$ (we only know $\mathcal{H}^{1}(E)<\infty$ because our assumption on $E$ ). However, as far as we know, all $T(b)$ type theorems require the estimate

$$
\left|\nu_{0}\right|(E) \leq C \mu(E)
$$

(and often stronger assumptions involving the $L^{\infty}$ norm of $b$ ). So by a direct application of a $T(b)$ type theorem we will obtain bad results when $\gamma(E) \ll \mathcal{H}^{1}(E)$.

To prove Theorem 1.1, we need to work with a measure "better behaved" than $\nu_{0}$, which we call $\nu$. This new measure will be a suitable modification of $\nu_{0}$ with the required estimate for its total variation. To construct $\nu$, we consider a set $F$ containing $E$ made up of a finite disjoint union of squares: $F=\bigcup_{i \in I} Q_{i}$. One should think that the squares $Q_{i}$ approximate $E$ at some "intermediate scale". For example, if $E=E_{N}$ is $N$-th approximation of the corner quarters Cantor set, then $F$ coincides with $E_{N / 2}$ (assuming $N$ even), and the squares $Q_{i}$ are the $4^{N / 2}$ squares of generation $N / 2$. For each square $Q_{i}$, we take a complex measure $\nu_{i}$ supported on $Q_{i}$ such that $\nu_{i}\left(Q_{i}\right)=\nu_{0}\left(Q_{i}\right)$ and $\left|\nu_{i}\right|\left(Q_{i}\right)=\left|\nu_{i}\left(Q_{i}\right)\right|$ (that is, $\nu_{i}$ will be a constant multiple of a positive measure). We set $\nu=\sum_{i} \nu_{i}$. Then, if the squares $Q_{i}$ are big enough, the variation $|\nu|$ will be sufficiently small. On the other hand, the squares $Q_{i}$ cannot be too big, because we will need

$$
\gamma_{+}(F) \leq C \gamma_{+}(E)
$$

In this way, we will have constructed a complex measure $\nu$ supported on $F$ satisfying

$$
|\nu|(F) \approx|\nu(F)|=\gamma(E) .
$$


Taking a suitable measure $\mu$ such that $\operatorname{supp}(\mu) \supset \operatorname{supp}(\nu)$ and $\mu(F) \approx \gamma(E)$, we will be ready for the application of a $T(b)$ type theorem. Indeed, notice that (4.8) implies that $\nu$ satisfies a global paraaccretivity condition and that also the variation $|\nu|$ is controlled. On the other hand, if we have been careful enough, we will have also some useful estimates on $|\mathcal{C} \nu|$, since $\nu$ is an approximation of $\nu_{0}$ (in fact, when defining $\nu$ in the paragraph above, the measures $\nu_{i}$ have to be constructed in a smoother way). Using a suitable $T(b)$ theorem, we will deduce $\gamma_{+}(F) \geq C^{-1} \mu(E)$, and so $\gamma_{+}(E) \geq C^{-1} \gamma(E)$, by (4.7), and we will be done. Nevertheless, as in the case of the Cantor sets $E(\lambda)$, in order to obtain the right estimates on the measures $\nu$ and $\mu$ it will be necessary to use an induction argument involving the sizes of the squares $Q_{i}$, which will allow to assume that $\gamma\left(E \cap Q_{i}\right) \approx \gamma_{+}\left(E \cap Q_{i}\right)$ for each square $Q_{i}$.

Now we will make some comments regarding the new difficulties that arise in the case of general sets. The choice of the right squares $Q_{i}$ which approximate $E$ at an intermediate scale is more complicated than in the case of the corner quarters Cantor set. The key estimates which the squares $Q_{i}$ must satisfy for the arguments above to work are the following:

$$
\gamma_{+}(F)=\gamma_{+}\left(\bigcup_{i} Q_{i}\right) \leq C \gamma_{+}(E)
$$

and

$$
\sum_{i} \gamma_{+}\left(Q_{i} \cap E\right) \leq C \gamma_{+}(E)
$$

For the corner quarters Cantor set, it can be checked easily that both (4.9) and (4.10) hold, using that $\gamma_{+}\left(E_{N}\right) \approx N^{-1 / 2}$ for all $N$.

Then, modulo some technicalities, we set $d \mu=\sum_{i} d s_{\mid \Gamma_{i}}$, where $\Gamma_{i}$ is a circumference of radius $\gamma\left(E \cap Q_{i}\right) / 10$ concentric with $Q_{i}$. We also define $d \nu=\sum_{i} a_{i} d s_{\mid \Gamma_{i}}$, with $a_{i}=\nu_{0}\left(Q_{i}\right) / \operatorname{length}\left(\Gamma_{i}\right)$.

In the construction of the squares $Q_{i}$ fulfilling (4.9) and (4.10), the characterization of $\gamma_{+}$in terms of curvature and the properties of the associated potential defined in (2.5) play an important role. Let $\sigma$ be a Radon measure which is maximal for the right hand side of (2.4) (and so for $\gamma_{+}$, in a sense). By variational arguments, it is not difficult to see that $\sigma(E) \approx \gamma_{+}(E)$ and that $U_{\sigma}(x) \geq \lambda_{0}$ for all $x \in E$, where $\lambda_{0}>0$ is some absolute constant. We consider the open set $\Omega=\left\{x \in \mathbb{C}: U_{\sigma}(x)>\varepsilon \lambda_{0}\right\}$, with $\varepsilon<10^{-3}$ fixed. Notice that $\Omega \subset E$ and

$$
\gamma_{+}(\Omega) \leq C\left(\varepsilon \lambda_{0}\right)^{-1} \sigma(E) \approx \gamma_{+}(E)
$$

by (2.7). Then, the squares $Q_{i}$ are precisely the Whitney squares of $\Omega$ which intersect $E$, and we set $F=\bigcup_{i} Q_{i}$.

Observe that (4.9) is a direct consequence of (4.11). 
The inequality (4.10) requires some additional arguments. It turns out that

$$
U_{\sigma_{\mid 2 Q_{i}}}(x)>\lambda_{0} / 10 \quad \text { for all } x \in Q_{i} \cap E,
$$

if $\varepsilon$ has been chosen small enough. This follows from the fact that there exists some point $x^{\prime} \notin \Omega$, with $\left|x-x^{\prime}\right| \approx \ell\left(Q_{i}\right)$, such that $U_{\sigma}\left(x^{\prime}\right) \leq \varepsilon \lambda_{0}$, which implies that $U_{\sigma_{\mid \mathbb{C} \backslash 2 Q_{i}}}(x) \ll \lambda_{0}$. Since $U_{\sigma}(x)>\lambda_{0}$, most of the potential $U_{\sigma}(x)$ must be originated by $\sigma_{\mid 2 Q_{i}}$, and then (4.12) holds. Thus, using (2.7) again and the fact that $\sum_{i} \chi_{2 Q_{i}} \leq C$, we get

$$
\sum_{i} \gamma_{+}\left(Q_{i} \cap E\right) \leq C \lambda_{0}^{-1} \sum_{i} \sigma\left(2 Q_{i}\right) \leq C \lambda_{0}^{-1} \sigma(E) \approx \gamma_{+}(E) .
$$

Another big difference between the proof for the Cantor sets $E(\lambda)$ and the proof for general compact sets comes from the $T(b)$ theorem. The corner quarters Cantor set, as well as the other Cantor sets $E(\lambda)$, have a "regular" structure which allows an easy application of the local $T(b)$ theorem of Christ. A general compact set does not have this nice structure. Further, the measure $\mu$ mentioned above is non doubling, in general. So a much more flexible $T(b)$ type theorem is needed. To this end, the $T(b)$ theorem of Nazarov, Treil and Volberg in [NTV] is a very powerful tool. The version of this theorem used in [To4] requires the following hypotheses:

- There exists a $\mu$-measurable function $b$ such that

(a) $\|b\|_{\infty} \leq C$,

(b) $\left\|\mathcal{C}_{*}(b d \mu)\right\|_{L^{1}(\mu)} \leq C \mu(F)$,

(c) $\mu(F) \leq C \int b d \mu$.

- Roughly speaking, there is a subset $G_{0} \subset F$ with $\mu\left(G_{0}\right) \approx \mu(F)$ where both $\mu$ has linear growth and $b$ is locally paraaccretive.

These assumptions ensure that there exists a subset $G \subset G_{0}$ such that the Cauchy transform is bounded on $L^{2}\left(\mu_{\mid G}\right)$. As a consequence, we deduce

$$
\gamma_{+}(E) \geq C^{-1} \gamma_{+}(F) \geq C^{-1} \mu(G) \approx \mu(F) \geq C^{-1} \gamma(E) .
$$

Of course, in this $T(b)$ theorem $\mu$ is allowed to be non doubling. Notice also that it only requires a control over the $L^{1}$ norm of $\mathcal{C}_{*}(b d \mu)$, instead of the usual $L^{\infty}$ or $B M O$ norms.

\section{Applications to rational and harmonic approximation}

In this section we will give some applications of the semiadditivity of analytic capacity to problems on uniform approximation of analytic functions by rational functions on compact sets, and also to questions on $\mathcal{C}^{1}$ approximation of harmonic functions on compact sets.

Let $X \subset \mathbb{C}$ be compact, and let $R(X)$ be the space of functions on $X$ which are uniform limits of analytic functions on $X$, or equivalently, which are uniform limits of rational functions with poles off $X$. Let $A(X)$ be the

algebra of those continuous functions on $X$ which are analytic on $\stackrel{\circ}{X}$. It is 
clear that $R(X) \subset A(X)$. We are interested in characterizing those compact sets $X$ for which $R(X)=A(X)$.

In order to explain the solution of this problem we need to introduce a slight variant of analytic capacity. The continuous analytic capacity of a compact set $X \subset \mathbb{C}$ is defined as

$$
\alpha(X)=\sup \left|f^{\prime}(\infty)\right|
$$

where the supremum is taken over all functions $f$ which are continuous in $\mathbb{C}$, analytic on $\mathbb{C} \backslash X$ and satisfy $|f(z)| \leq 1, z \in \mathbb{C}$. For a general set $F \subset \mathbb{C}$, we set $\alpha(F)=\sup \{\alpha(X): X \subset F$, X compact $\}$. Clearly, $\alpha \leq \gamma$. If $I \subset \mathbb{C}$ is an interval it is easy to check that $\alpha(I)=0$, and so $\alpha$ and $\gamma$ are different set functions. They coincide, however, on open sets.

In the 1960's Vitushkin introduced a powerful new method to deal with the above approximation problem leading to fairly complete solutions, thus culminating work previously done by Lavrentief, Mergelyan, Walsh and others. A good reference for this subject is the paper of Vitushkin [Vi]. We state now Vitushkin's Theorem.

TheOrem 5.1. For a compact set $X \subset \mathbb{C}$ the following are equivalent.

(a) $R(X)=A(X)$

(b) $\alpha(\Delta \backslash \stackrel{\circ}{X})=\gamma(\Delta \backslash X)$ for all open discs $\Delta \subset \mathbb{C}$.

(c) There exists $C>0$ such that $\alpha(\Delta \backslash \stackrel{\circ}{X}) \leq C \gamma(\Delta \backslash X)$ for all open discs $\Delta \subset \mathbb{C}$.

Conditions (a) and (b) must be interpreted as local negligibility conditions on $\partial X$ quantified by $\alpha$. Thus, the preceding problem on uniform rational approximation has been reduced to comparing the continuous analytic capacity of some sets.

The main drawback of Vitushkin's Theorem arises from the fact that conditions (b) and (c) above cannot be formulated in pure metric and/or geometric terms. As a consequence, there still remain many open problems in connection with the equality $R(X)=A(X)$. To state one of the most relevant, we need to introduce the notion of inner boundary of a compact set $X$.

The inner boundary $\partial_{i} X$ of $X$ is the set of boundary points which do not belong to the boundary of a connected component of $\mathbb{C} \backslash X$. Vitushkin showed that the equality $R(X)=A(X)$ essentially depends on the structure of $\partial_{i} X$ and he stated in $[\mathbf{V i}]$ the following conjecture:

$$
\text { If } \alpha\left(\partial_{i} X\right)=0 \text {, then } R(X)=A(X) \text {. }
$$

The first contribution to this question comes from the following result by Davie and Øksendal [DØ].

Theorem 5.2. If $\operatorname{dim}\left(\partial_{i} X\right)<1$, then $R(X)=A(X)$.

Here $\operatorname{dim}\left(\partial_{i} X\right)$ stands for the Hausdorff dimension of $\partial_{i} X$. Using Theorem 1.2 , this result can be improved as follows. 
Theorem 5.3. If $\gamma\left(\partial_{i} X\right)=0$, then $R(X)=A(X)$.

Proof. For each open disc $\Delta$, we set

$$
\Delta \backslash \stackrel{\circ}{X}=(\Delta \backslash X) \cup\left(\Delta \cap \partial_{e} X\right) \cup\left(\Delta \cap \partial_{i} X\right),
$$

where $\partial_{e} X=\partial X \backslash \partial_{i} X$ is the outer boundary of $X$. The arguments in the proof of Mergelyan's Theorem (see [Gam, Chapter VIII] or [Ve1]) can be adapted to show that $\alpha(\Delta \backslash \stackrel{\circ}{X})=\alpha\left((\Delta \backslash X) \cup\left(\Delta \cap \partial_{i} X\right)\right)$. Now, because of the semiadditivity of $\gamma$, we have

$$
\alpha(\Delta \backslash \stackrel{\circ}{X}) \leq C\left(\gamma(\Delta \backslash X)+\gamma\left(\Delta \cap \partial_{i} X\right)\right)=C \gamma(\Delta \backslash X)=C \alpha(\Delta \backslash X),
$$

since $\Delta \backslash X$ is open.

This result asserts, in particular, that if we take a compact set $X$, for which $\partial_{i} X$ is the corner quarters Cantor set, then $R(X)=A(X)$.

To solve the inner boundary conjecture it would be enough to prove the semiadditivity of continuous analytic capacity, namely that there exists a constant $C$ such that

$$
\alpha(E \cup F) \leq C(\alpha(E)+\alpha(F)) \text { for all Borel sets } E \text { and } F .
$$

Let us remark that this inequality does not seem to be an easy consequence of Theorem 1.2. The reader may obtain more information on this problem and related questions in [Dve].

Now we turn our attention to $\mathcal{C}^{1}$ approximation of harmonic functions. We will derive an analog of Mergelyan's Theorem (see [Gam, Chapter VIII] or $[\mathbf{V e 1}])$ for harmonic functions from Theorem 1.1. For $X \subset \mathbb{C}$ compact, we consider the space

$$
\mathcal{C}^{1}(X)=\mathcal{C}^{1}(\mathbb{C}) / J(X),
$$

endowed with the quotient norm, where

$$
J(X)=\left\{f \in \mathcal{C}^{1}(\mathbb{C}): f(z)=0 \text { and } \nabla f(z)=0, z \in X\right\} .
$$

The space $\mathcal{C}^{1}(\mathbb{C})$ is equipped with its natural Banach space structure given by the norm $\|f\|=\sum_{|\alpha| \leq 1}\left\|\partial^{\alpha} f\right\|_{\infty}$.

Whitney's extension Theorem identifies $\mathcal{C}^{1}(X)$ with a space of jets. A $\mathcal{C}^{1}$-jet is a triple $\left(f_{0}, f_{1}, f_{2}\right)$ of continuous functions on $X$ such that

$$
f_{0}(w)-f_{0}(z)-f_{1}(z)(w-z)-f_{2}(z)(\bar{w}-\bar{z})=o(|w-z|),
$$

the small $o$ being uniform in $z, w \in X$.

Set $H(X)=\left\{f \in \mathcal{C}^{1}(X): f_{0}\right.$ is harmonic on $\left.\stackrel{\circ}{X}\right\}$ and let $h(X)$ be the closure in $\mathcal{C}^{1}(X)$ of the functions in $\mathcal{C}^{1}(\mathbb{C})$ which are harmonic in a neighborhood of $X$. 
The $\mathcal{C}^{1}$ approximation problem for the Laplacian was studied by Paramonov $[\mathbf{P a}]$. The natural capacities associated to this problem are $\kappa^{\prime}$ and $\kappa$. Given a compact set $E \subset \mathbb{R}^{n}, \kappa^{\prime}(E)$ is defined as

$$
\kappa^{\prime}(E)=\sup |\langle T, 1\rangle|
$$

where the supremum is taken over all distributions $T$ supported on $E$ such that $\left\|\frac{x}{\|x\|^{n}} * T\right\|_{\infty} \leq 1$. The capacity $\kappa$ is the continuous version of $\kappa^{\prime}$. Its definition is analogous to the one of $\kappa^{\prime}$, with the additional condition that $\frac{x}{\|x\|^{n}} * T$ must be continuous. For $n=2, \kappa^{\prime}$ and $\kappa$ coincide with $\gamma_{\operatorname{Re}}$ and $\alpha_{\text {Re }}$, which are the versions of $\gamma$ and $\alpha$ originated by bounded Cauchy potentials of real measures.

Paramonov's result reads as follows.

TheOREM 5.4. For a compact set $X \subset \mathbb{C}$ the following are equivalent.

(a) $H(X)=h(X)$.

(b) There exists $C>0$ such that $\kappa(\Delta \backslash \stackrel{\circ}{X}) \leq C \kappa(\Delta \backslash X)$ for all open discs $\Delta \subset \mathbb{C}$.

Let $P(X)$ be the closure in $\mathcal{C}^{1}(X)$ of the space of harmonic polynomials in $\mathbb{C}$. The version of Mergelyan's Theorem for harmonic functions mentioned above (which was an open problem up to now) is the following.

Theorem 5.5. For a compact set $X \in \mathbb{C}, H(X)=P(X)$ if and only if $\mathbb{C} \backslash X$ is connected.

Proof. The necessity was proved in [Pa, Theorem 7.1]. To obtain the sufficiency we check that condition (b) of the preceding theorem is fulfilled for discs centered at the boundary of $X$. It can be shown that this condition already implies $H(X)=h(X)$.

From Theorem 1.1 we deduce that $\gamma(E) \approx \gamma_{\mathrm{Re}}(E)=\kappa^{\prime}(E)$ for any compact set $E \subset \mathbb{C}$. In particular, if $E$ is a continuum, then $\kappa^{\prime}(E) \approx$ $\operatorname{diam}(E)$. Suppose that $\Delta$ is a disc centered at some point in $\partial X$. Since $\Delta \backslash X$ contains a continuum $E$ of diameter comparable to the radius of $\Delta$, we have

$$
\kappa(\Delta \backslash X)=\kappa^{\prime}(\Delta \backslash X) \geq \kappa^{\prime}(E) \geq C^{-1} \operatorname{diam}(\Delta) \geq C^{-1} \kappa(\Delta \backslash \stackrel{\circ}{X}) .
$$

Thus, each function in $H(X)$ can be approximated by functions in $h(X)$. Then, using Walsh's analog of Runge's Theorem for harmonic functions [Wa] we obtain $H(X)=P(X)$.

\section{Open problems}

In this section we state several open problems related to the ones considered in the preceding sections. They are quite well known and, of course, there is no attempt of originality.

Problem 1. Is the continuous analytic capacity $\alpha$ semiadditive? This problem, raised by Vitushkin $[\mathbf{V i}]$, has already been mentioned at the end 
of Section 5. It has several applications to uniform rational approximation. In particular, it implies the inner boundary conjecture.

A closely related question is the comparability between $\alpha$ and $\alpha_{+}$, which is the version of $\alpha$ originated by Cauchy transforms of positive measures.

Problem 2. Is the class of sets with positive analytic capacity invariant under bilipschitz transformations? That is, if $E$ is compact, $\gamma(E)>0$ and $\varphi: \mathbb{C} \longrightarrow \mathbb{C}$ is bilipschitz, then can we claim that $\gamma(\varphi(E))>0$ ? From Theorem 1.1 and the characterization of $\gamma_{+}$in terms of curvature, it is quite easy to see that this is true if $\varphi \in \mathcal{C}^{1+\varepsilon}, \varepsilon>0$ (see [To1], for example). The analogous problem for $\varphi$ bilipschitz or $\mathcal{C}^{1}$ seems much more difficult.

Problem 3. The capacities $\kappa^{\prime}$ and $\kappa$ in $\mathbb{R}^{n}$. As mentioned in Section 5, these capacities were introduced by Paramonov $[\mathbf{P a}]$ to deal with problems of harmonic approximation in $\mathcal{C}^{1}$ norm. One can ask several questions about $\kappa^{\prime}$ and $\kappa$. For example, are they semiadditive? Are $\kappa^{\prime}$ and $\kappa$ comparable to their corresponding positive versions $\kappa_{+}^{\prime}$ and $\kappa_{+}$? The main obstacle that appears when one tries to extend the methods used to prove the semiadditivity of analytic capacity is the absence of a notion such as the Menger curvature of a measure (see $[\mathbf{F a}]$ ), which plays a key role in the proof of Theorem 1.1, as shown in Section 4.

Problem 4. Riesz transforms and uniform rectifiability. A set $E \subset \mathbb{R}^{n}$ is called AD-regular of dimension $d$ if

$$
\mathcal{H}^{d}(E \cap B(x, r)) \approx r^{d} \quad \text { for all } x \in E \text { and } 0<r \leq \operatorname{diam}(E),
$$

where $\mathcal{H}^{d}$ stands for the $d$-dimensional Hausdorff measure. In [MMV] it has been proved that if $E \subset \mathbb{C}$ is an AD-regular 1-dimensional set such that the Cauchy transform is bounded on $L^{2}\left(\mathcal{H}_{\mid E}^{1}\right)$, then $E$ is contained in an AD-regular curve.

In the corresponding $d$-dimensional (with $d>1$ ) version of the problem, one has a set $E \subset \mathbb{R}^{n}$ and it is assumed that the $d$-dimensional Riesz transforms are bounded on $L^{2}\left(\mathcal{H}_{\mid E}^{d}\right)$. Then one asks if $E$ is uniformly rectifiable in the sense of David and Semmes [DS]. This problem still remains open. David and Semmes [DS] have proved it under the stronger assumption of the $L^{2}$ boundedness of all Calderón-Zygmund operators with an odd kernel which is $\mathcal{C}^{\infty}$ away from the origin (see also [DS] for other partial results). As in the preceding problem, the main difference with the 1-dimensional case comes from the absence of a notion similar to Menger curvature, which relates analytic concepts, such as $L^{2}$ boundedness, with metric-geometric information via a formula like (2.2).

\section{References}

[Ah] L. Ahlfors, Bounded analytic functions, Duke Math. J. 14 (1947), 1-11. 
[Ch1] M. Christ, A T (b) theorem with remarks on analytic capacity and the Cauchy integral. Colloq. Math. 60/61(2) (1990), 601-628.

[Ch2] M. Christ, Lectures on Singular Integral Operators. CBMS Regional Conf. Ser. in Math. 77, Amer. Math. Soc., Providence, 1990.

[CW] R.R. Coifman and G. Weiss, Analyse harmonique non-commutative sur cetains spaces homogènes. Lecture Notes in Math. 242, Springer-Verlag, Berlin, 1971.

[Da] G. David, Unrectifiable 1-sets have vanishing analytic capacity. Revista Mat. Iberoamericana 14(2) (1998), 369-479.

[DS] G. David and S. Semmes, Analysis of and on uniformly rectifiable sets. Mathematical Surveys and Monographs, 38. American Mathematical Society, Providence, RI, 1993.

[Dve] A.M. Davie, Analytic capacity and approximation problems. Trans. Amer. Math. Soc. 171 (1972), 409-444.

[DØ] A.M. Davie and B. Øksendal, Analytic capacity and differentiability properties of finely harmonic functions. Acta Math. 149 (1982),127-152.

[Ei] V. Eiderman, Hausdorff measure and capacity associated with Cauchy potentials. (Russian) Mat. Zametki 63 (1998), 923-934. Translation in Math. Notes 63 (1998), 813-822.

[Fa] H.M. Farag, The Riesz kernels do not give rise to higher-dimensional analogues of the Menger-Melnikov curvature. Publ. Mat. 43 (1999), no. 1, 251-260.

[Gam] T.W. Gamelin, Uniform Algebras. Prentice Hall, Englewood Cliffs, NJ, 1969.

[Gar1] J. Garnett, Positive length but zero analytic capacity. Proc. Amer. Math. Soc. 24 (1970), 696-699.

[Gar2] J. Garnett, Analytic capacity and measure. Lecture Notes in Math. 297, SpringerVerlag, 1972.

[MTV] J. Mateu, X. Tolsa and J. Verdera, The planar Cantor sets of zero analytic capacity and the local T(b)-Theorem. Preprint (2001). To appear in J. Amer. Math. Soc.

[Ma] P. Mattila, On the analytic capacity and curvature of some Cantor sets with non$\sigma$-finite length. Publ. Mat. 40 (1996), 127-136.

[MMV] P. Mattila, M.S. Melnikov and J. Verdera, The Cauchy integral, analytic capacity, and uniform rectifiability. Ann. of Math. (2) 144 (1996), 127-136.

[Me] M.S. Melnikov, Analytic capacity: discrete approach and curvature of a measure. Sbornik: Mathematics 186(6) (1995), 827-846.

[MV] M.S. Melnikov and J. Verdera, A geometric proof of the $L^{2}$ boundedness of the Cauchy integral on Lipschitz graphs. Internat. Math. Res. Notices (1995), 325-331.

[NTV] F. Nazarov, S. Treil and A. Volberg, How to prove Vitushkin's conjecture by pulling ourselves up by the hair. Preprint (1997).

[Pa] P.V. Paramonov, On harmonic approximations in the $C^{1}$-norm. (Russian) Mat. Sb. 181 (1990), no. 10, 1341-1365; translation in Math. USSR-Sb. 71 (1992), no. 1, 183-207.

[To1] X. Tolsa, Curvature of measures, Cauchy singular integral and analytic capacity. Ph. D. Thesis, Universitat Autònoma de Barcelona, 1998.

[To2] X. Tolsa, $L^{2}$-boundedness of the Cauchy integral operator for continuous measures. Duke Math. J. 98(2) (1999), 269-304.

[To3] X. Tolsa, On the analytic capacity $\gamma_{+}$. Indiana Math. J. 51(2) (2002), 317-343.

[To4] X. Tolsa, Painlevé's problem and the semiadditivity of analytic capacity. Preprint (2001). To appear in Acta Math.

[Ve1] J. Verdera, Removability, capacity and approximation. In "Complex Potential Theory", (Montreal, PQ, 1993), NATO Adv. Sci. Int. Ser. C Math. Phys. Sci. 439, Kluwer Academic Publ., Dordrecht, 1994, pp. 419-473.

[Ve2] J. Verdera, On the T(1)-theorem for the Cauchy integral. Ark. Mat. 38 (2000), 183-199. 
[Ve3] J. Verdera, $L^{2}$ boundedness of the Cauchy integral and Menger curvature. Contemp. Math. 277 (2001), 139-158.

[Vi] A. G. Vitushkin, The analytic capacity of sets in problems of approximation theory. Uspeikhi Mat. Nauk. 22(6) (1967), 141-199 (Russian); in Russian Math. Surveys 22 (1967), 139-200.

[Wa] J.L. Walsh, The approximation of harmonic functions by harmonic polynomials and by harmonic rational functions. Bull. Amer. Math. Soc. 35 (1929), 499-544.

Departament de Matemàtiques, Universitat Autònoma de Barcelona, 08193 Bellaterra (BARCElona), Spain

E-mail address: mateu@mat.uab.es

E-mail address: xtolsa@mat.uab.es

E-mail address: jvm@mat.uab.es 